\title{
Low surgical Apgar score in older patients with gynecological cancer is a risk factor for postoperative complications and 1-year mortality: A multicenter retrospective cohort study
}

\author{
MAKOTO YAMAMOTO $^{1}$, KAZUMI KURATA ${ }^{1}$, MIKIKO ASAI-SATO ${ }^{2}$, MAYU SHIOMI ${ }^{3}$, \\ YUTAKA UEDA $^{3}$, YOICHI AOKI ${ }^{4}$ and YOSHIO YOSHIDA ${ }^{1}$
}

\author{
${ }^{1}$ Department of Obstetrics and Gynecology, Faculty of Medical Sciences, University of Fukui, Fukui 910-1193; \\ ${ }^{2}$ Department of Obstetrics and Gynecology, Yokohama City University Graduate School of Medicine, \\ Kanagawa 236-0004; ${ }^{3}$ Department of Obstetrics and Gynecology, Osaka University Graduate School of \\ Medicine, Osaka 565-0871; ${ }^{4}$ Department of Obstetrics and Gynecology, Graduate School of Medicine, \\ University of The Ryukyus, Okinawa 903-0215, Japan
}

Received June 24, 2020; Accepted October 14, 2020

DOI: $10.3892 /$ mco.2020.2183

\begin{abstract}
Little is known about preoperative and intraoperative risk factors for postoperative complications in older patients with gynecological cancer. The present retrospective multi-institutional study included 173 older patients with primary gynecological cancer between January 2015 and December 2015 at four institutions. The cancer stage, medical history, Charlson comorbidity score, body mass index (BMI), subjective global assessment, fall risk assessment, American Society of Anesthesiologists physical status classification, surgical Apgar score (SAS), type of surgery and 1-year postoperative mortality were investigated. Multivariate analysis revealed that BMI and mental illness were risk factors for postoperative complications, and low SAS increased the risk for both postoperative complications and mortality within 1 year. Receiver operating characteristic curve analysis of postoperative complications in terms of SAS revealed that low SAS predicted high risk with a sensitivity of $85.7 \%$ and a specificity of $46.5 \%$, and high SAS predicted low risk with a sensitivity of $21.4 \%$ and a specificity of $95 \%$. The present results suggest that SAS, which is an intraoperative assessment, may be useful for assessing the risks of postoperative complications and mortality within 1 year. It is important to develop a preoperative assessment tool that can predict a low SAS score and reflect the postoperative prognosis of older patients with gynecological cancer.
\end{abstract}

Correspondence to: Dr Yoshio Yoshida, Department of Obstetrics and Gynecology, Faculty of Medical Sciences, University of Fukui, 23-3 Matsuoka-Shimoaizuki, Eiheiji-cho, Yoshida-gun, Fukui 910-1193, Japan

E-mail: yyoshida@u-fukui.ac.jp

Key words: fit, vulnerable, frail, geriatric assessment, surgical Apgar score

\section{Introduction}

In Japan, the elderly comprised $27.7 \%$ of the total population in 2017, making it the world's most rapidly aging society. Moreover, 19.89 million women in Japan are aged 65 years or over and account for $30.6 \%$ of the female population. By 2040, the elderly populations of other Asian countries such as South Korea, Singapore, and Thailand are predicted to reach levels similar to the current level in Japan (1).

Given this background, gynecologists are expected to treat an increasing number of older patients in the future. Among the elderly, there is wide individual variation in health status, ranging from robust individuals to frail elderly with serious underlying conditions. Frail individuals who develop gynecological cancer are often treated surgically, but postoperative complications may arise and affect their activities of daily living. Greater efforts to reduce such complications are required on the part of gynecologists. However, sufficient evidence supporting the prediction of elderly gynecological patients who are more likely to develop postoperative complications has not yet been obtained.

During assessment of the indications for surgery, gynecologists often rely on ambiguous methodology, thereby risking under- or over-treatment. Preoperative frailty assessments have been useful for predicting postoperative complications, but these have been conducted in a variety of ways and no standard assessment has been established. This multicenter study was conducted to identify the preoperative and intraoperative risk factors for postoperative complications in older patients with gynecological cancer. In this study, we focused on postoperative complications and short-term prognosis.

\section{Materials and methods}

Patients. The study subjects included elderly patients aged $\geq 65$ years who were diagnosed with primary gynecological cancer between January 2015 and December 2015 at four Japanese university hospitals located in major cities (Osaka 
University Hospital and Yokohama City University Hospital) and in rural areas (Ryukyu University Hospital and Fukui University Hospital). Age, family composition, number of children, type of cancer, cancer stage, medical history, Charlson comorbidity score (CCS), body mass index (BMI), subjective global assessment (SGA), fall risk assessment, American Society of Anesthesiologists physical status classification, surgical Apgar score (SAS), type of surgery, and 1-year postoperative mortality were investigated retrospectively and compared between subjects who did and did not develop complications.

The SAS, which was developed by Gawande et al (2) for patients undergoing open colectomy, is an index for assessing a patient's general condition during surgery and has been shown to be useful for predicting complications after general and vascular surgery. Similar to the Apgar score used for neonatal evaluation at birth, the SAS is scored out of 10 , with a higher score indicating a better condition. It is very simple to use, and evaluates patients on the basis of the three parameters of estimated blood loss, lowest mean blood pressure, and lowest heart rate. It can be calculated postoperatively from anesthesia records. One tool for assessing the probability of postoperative complications is the web-based National Surgical Quality Improvement Program Surgical Risk Calculator, which was developed by the American College of Surgeons (3) and can show the percentage probability of the occurrence of serious complications for each parameter. Although it has allowed patients to understand the assessment results more easily, it has been reported by some studies to not be particularly well suited to gynecological cancer surgery $(4,5)$.

Family composition was classified as living with others, living alone, or other, such as institutional resident or unknown. The type of cancer was classified as cervical cancer, uterine cancer, uterine sarcoma, ovarian cancer, or vaginal/vulvar cancer. Staging was according to the International Federation of Gynecology and Obstetrics clinical staging system or the surgical staging system (6-8). In the medical history, hypertension was defined as a patient who had undergone medical treatment, excluding cases that were treated only with diet or exercise. Coronary artery disease was defined as a definitive diagnosis obtained by cardiac catheterization or other testing. Arrhythmia was defined as present only if the patient had undergone medical treatment. Heart dysfunction was defined as grade 2 or worse aortic valve insufficiency, aortic stenosis, mitral insufficiency, or tricuspid insufficiency, as confirmed by echocardiography, excluding grade 1 conditions that did not affect daily life. Cerebrovascular disorder was defined as noted in the medical records. Rheumatoid arthritis was defined as treatment with antirheumatic drugs. Mental illness was defined as schizophrenia, major depressive disorder, or a similar disorder that had been diagnosed and treated with oral psychotropic agents, excluding treatment only with sleep medications. Dementia was defined as noted in the patient's medical history, regardless of oral medication intake. Asthma and dyslipidemia were defined as noted in the patient's medical history. Diabetes mellitus was defined as treatment with oral medication or insulin, excluding treatment only with diet or exercise. Osteoporosis was defined as noted in the patient's medical history, based on treatment that had been provided, or when strongly suspected due to femoral neck fracture or a similar event in the patient's clinical history.

The CCS was formulated as a preoperative comorbidity score in 1987 by Charlson et al (9). In the present study, the version revised by Quan et al (10) in 2011 was used. The CCS classifies patients according to the weight of 12 different medical conditions, with a higher score indicating more severe comorbidities. The BMI was classified as normal or obese, based on a cutoff value of $25 \mathrm{~kg} / \mathrm{m}^{2}$. Using the malnutrition risk score upon hospital admission, the SGA was scored on a 3-point scale as low (1), moderate (2), or high (3). The postoperative fall risk was assessed using somewhat different criteria among the different institutions, but an assessment that broadly conformed to the Fall Assessment Score Sheet, which was included in the Medical Safety Manual for Healthcare Professionals (11) published by the Japan Medical Association, was used.

As a measure of the patient's general condition during surgery, the SAS was calculated on the basis of estimated blood loss, lowest mean blood pressure, and lowest heart rate. Similar to the neonatal evaluation at birth, it was scored out of 10, with a higher score indicating a better condition (Table I). Receiver operator characteristic (ROC) curve analysis was performed to assess the ability of the SAS to predict postoperative complications in the elderly; the determined SAS cutoff values were used to divide the patients into 3 groups, including high risk (8-10), moderate risk (4-7), and low risk (0-3) for postoperative complications.

Postoperative complications were analyzed using the Clavien-Dindo classification, following the Japan Clinical Oncology Group criteria for postoperative complications (12). Complications were defined as Clavien-Dindo classification grade III or above and requirement for surgical, endoscopic, or interventional radiology treatment or intensive care. Cases that improved with medication alone were excluded.

Statistical analysis. Statistical analysis was performed using Fisher's exact test for comparisons between the 2 groups, and logistic regression analysis was performed for univariate and multivariate analysis. A P-value $<0.05$ was considered to indicate statistical significance. Sensitivity and specificity were evaluated using ROC curves. All data were analyzed using SPSS version 21 statistical software (IBM Inc., Armonk, NY, USA).

\section{Results}

Patients' characteristics. The patients' characteristics are shown in Table II. A total of 173 patients (mean age 72.54 years) were enrolled from the four institutions. Overall, $76.3 \%$ of the patients were living with their husband or other family members, and $87.3 \%$ had children.

The most common types of cancer were endometrial cancer $(46.8 \%)$ and ovarian cancer $(28.9 \%)$, both of which are increasing in Japan. In $62.4 \%$ of cases, the cancer was identified as stage I. The medical history of many patients included hypertension (46.2\%), dyslipidemia (26.0\%), and diabetes $(14.5 \%)$, all of which are lifestyle diseases. The incidence of dementia was $1.2 \%$, which was significantly lower than the estimated prevalence of $17.9 \%$ among Japanese 
Table I. Surgical Apgar score.

\begin{tabular}{lcccc}
\hline & \multicolumn{3}{c}{ Score } \\
\cline { 2 - 5 } Variable & 0 & 1 & 2 & 3 \\
\hline Intraoperative estimated amount of bleeding, ml & $>1,000$ & $601-1,000$ & $101-600$ & $\leq 100$ \\
Minimal mean arterial blood pressure, mmHg & $<40$ & $40-54$ & $55-69$ & $\geq 70$ \\
Minimum heart rate, beats/minutes & $>85$ & $76-85$ & $66-75$ & $56-65$ \\
\hline
\end{tabular}

Table II. Patient characteristics.

\begin{tabular}{|c|c|}
\hline Characteristic & Value \\
\hline Number & 173 \\
\hline Age, years (mean \pm SD) & $72.54 \pm 6.289$ \\
\hline \multicolumn{2}{|l|}{ Family composition, n (\%) } \\
\hline Living alone & $41(23.7)$ \\
\hline Living with others & $102(59.0)$ \\
\hline Other & $30(17.3)$ \\
\hline \multicolumn{2}{|l|}{ Children, n (\%) } \\
\hline No & $21(12.1)$ \\
\hline Yes & $151(87.3)$ \\
\hline Unknown & $1(0.6)$ \\
\hline Cervical cancer, n (\%) & $16(9.2)$ \\
\hline Endometrial cancer, n (\%) & $81(46.8)$ \\
\hline Uterine sarcoma, n (\%) & $12(6.9)$ \\
\hline Ovarian cancer, n (\%) & $50(28.9)$ \\
\hline Vaginal/vulvar cancer, n (\%) & $14(8.1)$ \\
\hline \multicolumn{2}{|l|}{ Stage, n (\%) } \\
\hline I & $108(62.4)$ \\
\hline II & $15(8.7)$ \\
\hline III & $38(22.0)$ \\
\hline IV & $11(6.4)$ \\
\hline Metastatic & $1(0.6)$ \\
\hline \multicolumn{2}{|l|}{ Surgical procedure, n (\%) } \\
\hline No open surgery & $11(6.4)$ \\
\hline $\begin{array}{l}\text { Open surgery without retroperitoneal } \\
\text { operations }\end{array}$ & $80(46.2)$ \\
\hline Open surgery with retroperitoneal operations & $82(47.4)$ \\
\hline \multicolumn{2}{|l|}{ G3 or worse complications, n (\%) } \\
\hline No & $159(91.9)$ \\
\hline Yes & $14(8.1)$ \\
\hline \multicolumn{2}{|l|}{ Death within 1 year postoperatively, n (\%) } \\
\hline No & $148(85.5)$ \\
\hline Yes & $10(5.8)$ \\
\hline Unknown & $15(8.7)$ \\
\hline
\end{tabular}

people aged $\geq 65$ years in 2012 . This was most likely because of the presence of undiagnosed cases or because surgery was not indicated for patients with dementia. Of those patients who underwent abdominal surgery, the number of cases who underwent retroperitoneal surgery was about the same as the number who did not (46.2 vs. $47.4 \%$ ). Ten patients (5.8\%) who underwent surgery died within 1 year postoperatively.

Postoperative prognosis. Grade 3 or worse postoperative complications occurred in 14 patients (8.1\%). There was 1 case of grade 5 complication of postoperative death caused by sepsis. Postoperative death within 1 year was significantly more frequent in patients with postoperative complications than in those without postoperative complications (Table III). Univariate analysis was carried out to investigate the parameters associated with postoperative complications and death within 1 year postoperatively. On univariate analysis (Table IV), BMI, mental illness, and SAS were significantly associated with postoperative complications, whereas cancer stage, CSS, and SAS were significantly associated with death within 1 year postoperatively. Multivariate analysis by logistic regression (Table $\mathrm{V}$ ) revealed that BMI and mental illness were risk factors for postoperative complications, whereas low SAS increased the risk for both postoperative complications and death within 1 year postoperatively.

Surgical apgar score. The number and percentage of patients in each SAS score are shown in Table VI. ROC analysis of the SAS for postoperative complications showed an area under the curve (AUC) of $0.649(\mathrm{P}=0.016)$. An SAS of $\leq 6$ points predicted postoperative complications with a sensitivity of $64.3 \%$ and specificity of $64.2 \%$. The SAS risk classification predicted high risk with sensitivity of $85.7 \%$ and specificity of $46.5 \%$ and low risk with sensitivity of $21.4 \%$ and specificity of 95\% (Fig. 1).

\section{Discussion}

In the present study, older gynecological cancer patients with a preoperative history of mental illness, high CCS, obesity, or low intraoperative SAS were found to be more likely to develop postoperative complications, and patients with a low SAS and those who developed postoperative complications were more likely to die within 1 year postoperatively. In particular, low SAS was an independent risk factor not only for the development of postoperative complications, but also for death within 1 year postoperatively.

A previous study reported that the lower the SAS, the higher the probability of major complications or death (2). Subsequently, the SAS was used in a large-scale study (13) and for gynecological surgery $(14,15)$. In a previous single-center study, we found that intraoperative SAS was useful for 
Table III. Association between postoperative complications and death within 1 year postoperatively.

\begin{tabular}{|c|c|c|c|c|}
\hline \multirow[b]{2}{*}{ G3 or worse postoperative complications } & \multicolumn{2}{|c|}{$\begin{array}{c}\text { Death within } 1 \text { year } \\
\text { postoperatively }\end{array}$} & \multirow[b]{2}{*}{ Total, $\mathrm{n}$} & \multirow[b]{2}{*}{ P-value } \\
\hline & No, $n$ & Yes, $\mathrm{n}$ & & \\
\hline No & 139 & 7 & 146 & $0.030^{\mathrm{a}}$ \\
\hline Yes & 9 & 3 & 12 & \\
\hline Total & 148 & 10 & 158 & \\
\hline
\end{tabular}

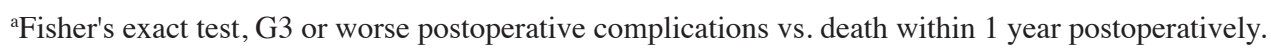

Table IV. Univariate analysis.

\begin{tabular}{|c|c|c|c|c|c|}
\hline \multirow[b]{2}{*}{ Variable } & \multirow[b]{2}{*}{ Value } & \multicolumn{2}{|c|}{$\begin{array}{l}\text { G3 or worse } \\
\text { complications }\end{array}$} & \multicolumn{2}{|c|}{$\begin{array}{c}\text { Death within } 1 \text { year } \\
\text { postoperatively }\end{array}$} \\
\hline & & P-value & Odds ratio $(95 \% \mathrm{CI})$ & P-value & Odds ratio $(95 \% \mathrm{CI})$ \\
\hline \multicolumn{6}{|l|}{ Patient characteristics } \\
\hline Age, years (mean $\pm \mathrm{SD})$ & $72.54 \pm 6.289$ & 0.91 & $1.00(0.91-1.09)$ & 0.24 & $1.06(0.96-1.17)$ \\
\hline Children, n (\%) & $21(12.1)$ & 0.98 & $1.01(0.23-15.19)$ & 0.98 & $0.97(0.11-8.26)$ \\
\hline Obesity (BMI $\geq 25 \mathrm{~kg} / \mathrm{m}^{2}$ ) & & 0.03 & $3.54(1.16-10.78)$ & 0.29 & $0.32(0.04-2.62)$ \\
\hline Living with other family members & & 0.46 & $2.53(0.22-29.29)$ & 0.15 & $8.75(0.45-168.60)$ \\
\hline \multicolumn{6}{|l|}{ Medical history, prevalence (\%) } \\
\hline Hypertension & $80(46.2)$ & 0.40 & $1.61(0.54-4.86)$ & 0.39 & $1.77(0.478-6.51)$ \\
\hline Coronary artery disease & $11(6.4)$ & $>0.99$ & - & $>0.99$ & - \\
\hline Arrhythmia & $11(6.4)$ & $>0.99$ & - & 0.47 & $2.24(0.248-20.22)$ \\
\hline Cardiac dysfunction & $12(6.9)$ & 0.98 & $1.04(0.12-8.66)$ & 0.47 & $2.24(0.248-20.22)$ \\
\hline Cerebrovascular impairment & $15(8.7)$ & $>0.99$ & - & 0.22 & $2.83(0.54-14.87)$ \\
\hline Rheumatoid arthritis & $1(0.6)$ & $>0.99$ & - & $>0.99$ & - \\
\hline Mental illness & $5(2.9)$ & 0.03 & $8.67(1.32-56.97)$ & 0.24 & $4.00(0.40-39.60)$ \\
\hline Dementia & $2(1.2)$ & $>0.99$ & - & $>0.99$ & - \\
\hline Asthma & $8(4.6)$ & 0.64 & $1.67(0.19-14.64)$ & $>0.99$ & - \\
\hline Diabetes & $25(14.5)$ & 0.99 & $0.99(0.21-4.69)$ & 0.71 & $0.67(0.08-5.58)$ \\
\hline Dyslipidemia & $45(26.0)$ & 0.82 & $1.15(0.34-3.87)$ & 0.38 & $1.80(0.48-6.71)$ \\
\hline Osteoporosis & $14(8.1)$ & 0.89 & $0.86(0.11-7.14)$ & $>0.99$ & - \\
\hline Stage ( 1 or 2 vs. 3 or 4$)$ & & 0.24 & $2.01(0.69-6.12)$ & 0.01 & $7.47(1.83-30.42)$ \\
\hline Surgical procedure (retroperitoneal operations) & & 0.73 & $0.86(0.35-2.07)$ & 0.12 & $0.70(0.26-1.87)$ \\
\hline \multicolumn{6}{|l|}{ Assessment score, mean \pm SD } \\
\hline CCS sore & $2.54 \pm 1.45$ & 0.40 & $1.14(0.84-1.54)$ & 0.01 & $1.43(1.10-1.86)$ \\
\hline SGA score & $1.10 \pm 0.40$ & 0.22 & $2.14(0.63-7.25)$ & 0.07 & $0.29(0.93-12.53)$ \\
\hline Fall risk assessment & $1.61 \pm 0.56$ & 0.08 & $0.37(0.13-1.12)$ & 0.06 & $0.27(0.07-1.07)$ \\
\hline ASA preoperative risk assessment & $1.88 \pm 0.53$ & 0.84 & $0.90(0.32-2.54)$ & 0.16 & $2.39(0.71-8.07)$ \\
\hline SAS score & $6.83 \pm 1.96$ & 0.02 & $0.72(0.55-0.94)$ & 0.01 & $0.64(0.46-0.89)$ \\
\hline
\end{tabular}

BMI, body mass index; CSS, Charlson comorbidity score; SGA, Subjective Global Assessment; ASA, American Society of Anesthesiologists; SAS, surgical Apgar score; SD, standard deviation; CI, confidence interval.

predicting postoperative complications in gynecological patients, including those without cancer (16). Moreover, the World Health Organization recommended the use of the SAS for safe surgery guidelines in the World Alliance for Patient Safety documents (17). The SAS cannot be used to assess the capability of patients to withstand surgery, because it is based on intraoperative data; however, it may be helpful in determining the requirement for postoperative intensive care. Based on our ROC analysis, high-risk patients had a high probability of requiring treatment for postoperative complications, 
Table V. Multivariate analysis using logistic regression analysis.

\begin{tabular}{lccccc}
\hline & \multicolumn{2}{c}{ G3 or worse complications } & & \multicolumn{2}{c}{ Death within 1 year postoperatively } \\
\cline { 2 - 3 } Variable & P-value & Odds ratio (95\% CI) & & P-value & Odds ratio (95\% CI) \\
\hline SAS score & 0.04 & $0.74(0.55-0.99)$ & & 0.05 & $0.69(0.47-0.99)$ \\
Obesity (BMI $\left.\geq 25 \mathrm{~kg} / \mathrm{m}^{2}\right)$ & 0.02 & $4.35(1.27-14.88)$ & & 0.56 & $0.52(0.06-4.68)$ \\
CCS score & 0.87 & $1.03(0.72-1.48)$ & & 0.23 & $1.21(0.89-1.66)$ \\
Mental illness & 0.02 & $11.68(1.43-95.48)$ & & 0.47 & $2.96(0.16-55.88)$ \\
Stage 1 or 2 vs. 3 or 4 & 0.45 & $1.69(0.43-6.64)$ & & 0.11 & $3.65(0.75-17.76)$ \\
\hline
\end{tabular}

SAS, surgical Apgar score; BMI, body mass index; CSS, Charlson comorbidity score.

Table VI. Number and percentage of patients with each SAS.

\begin{tabular}{ccc}
\hline SAS score & Number & Percentage \\
\hline 1 & 1 & 0.6 \\
2 & 3 & 1.7 \\
3 & 7 & 4.0 \\
4 & 14 & 8.1 \\
5 & 19 & 11.0 \\
6 & 22 & 12.7 \\
7 & 31 & 17.9 \\
8 & 36 & 20.8 \\
9 & 35 & 20.2 \\
10 & 5 & 2.9 \\
\hline
\end{tabular}

SAS, surgical Apgar score.

whereas low-risk patients had $>95 \%$ probability of not developing postoperative complications. Although the present study did not distinguish between open and laparoscopic surgery, it is important to note that SAS was originally developed for use in open surgery. Moreover, attempts have been made to determine the utility of the SAS for predicting perioperative complications during robotic-assisted radical hysterectomy, in which blood loss is low (18). Especially in endometrial cancer, the usefulness of minimally invasive surgery in the elderly has been reported $(19,20)$, and it is necessary to examine the usefulness of SAS in minimally invasive surgery in the future.

In the present study, history of mental illness, obesity, and low SAS were all risk factors for developing postoperative complications. Even a serious mental illness may not always be detected on standard preoperative tests, such as blood tests, urinalysis, electrocardiography, and plain radiography. Therefore, clinicians are likely to be unaware that such patients are at high risk. However, patients with psychiatric disorders tend to complain less actively about their symptoms because of the nature of their illness. Furthermore, the analgesic and sedative effects of psychotropic medications may have led these patients to have higher pain thresholds and to be less aware of postoperative abnormalities. In fact, around half of patients who were less likely to seek medical attention were reported to have physical disorders, and many cases were not

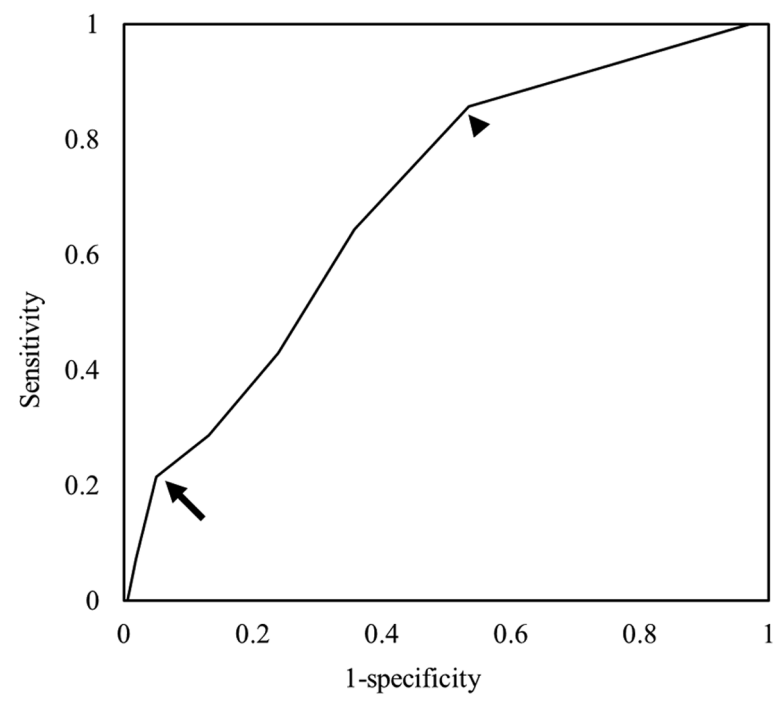

Figure 1. Receiver operating characteristic curve analysis of the surgical Apgar score for postoperative complications (area under the curve, 0.694; $\mathrm{P}=0.016)$. The cut-off points are shown for the high- and moderate-risk groups (arrowhead) and for the moderate- and low-risk groups (arrow).

correctly diagnosed (21). Patients on psychotropic medications were reported to have a high risk for venous thromboembolism (VTE) $(22,23)$, and those with schizophrenia were known to have an increased risk for not only VTE, but also respiratory failure (24). In a pooled analysis of 3 GINECO phase 2 trials by Tinquaut et al (25), depression and the Hospital Anxiety and Depression Scale score were associated with survival. In the present study, although mental illness was not found to have a direct association with death within 1 year postoperatively, it increased the risk for postoperative complications. These results suggest that the presence of mental illness in older patients with a gynecological malignancy may be associated with resistance to treatment. It is important to note that the present study defined postoperative complications as those with grade III or higher Clavien-Dindo classification, thereby excluding medically treated postoperative complications such as delirium. Therefore, the incidence of complications in patients with mental illness may have been underestimated. Clinicians should manage such patients with greater caution. To the best of our knowledge, no study has identified the association of postoperative complications with widely 
used antidepressants, such as selective serotonin reuptake inhibitors, serotonin-norepinephrine reuptake inhibitors, or noradrenergic and specific serotonergic antidepressants. This is an important topic for future research.

As previously mentioned, the present study showed that patients with low SAS and those who developed postoperative complications were more likely to die within 1 year postoperatively. However, the direct causality between postoperative complications and increased risk for death, as well as the possible confounding effect of a poor general condition on death within 1 year as a result of postoperative complications, was unclear. Nevertheless, because our analysis showed that SAS was an independent risk factor for death within 1 year postoperatively, patients with low SAS scores may require careful postoperative follow-up. Of the 3 patients who developed postoperative complications and died within 1 year, 1 patient was classified as CCS 2, SAS 3, and did not have a mental illness; 1 patient was CCS 2 , SAS 4, and did not have a mental illness; and 1 patient was CCS 9, SAS 5, and had a mental illness.

Based on our results, SAS may be useful for assessing the risks for postoperative complications and death within 1 year postoperatively. In the present study, the only predictor of death within 1 year postoperatively was the SAS score, which is an intraoperative assessment, and no preoperative predictive factor was identified. The Vulnerable Elders Survey-13 (VE-13) has been shown to be useful for pre-chemotherapy assessment (26), but other studies have found that the preoperative Comprehensive Geriatric Assessment and GA-GYN scores were not predictive of postoperative outcomes $(27,28)$. Therefore, a preoperative risk assessment score for the treatment of older patients with gynecological cancer has yet to be established. New preoperative assessment tools that can anticipate low SAS scores and reflect the postoperative prognosis of older gynecologic patients must be addressed and developed.

This study had a number of limitations. First, this study did not collect data on postoperative treatment. However, this study analyzes the short-term prognosis of one year, and we believe that the impact of the choice of postoperative treatment on the prognosis is limited. Although this was a multicenter analysis, it included only 173 patients from four hospitals. More gynecological institutions in Japan need to take part in clinical studies. In addition, alternative questions were used in the survey about postoperative complications. Therefore, in the 14 patients who developed postoperative complications, the detailed breakdown of conditions, such as infection, ileus, and thrombosis, was unknown. With the expected rise in the number of older patients who require treatment for gynecological cancer, the effects of newer psychotropic medications and the increasing use of laparoscopic surgery necessitate further study.

In conclusion, the SAS may be useful for assessing the risks of postoperative complications and death within 1 year postoperatively. It is important to develop a preoperative assessment tool that can predict a low SAS score and reflect the postoperative prognosis of older gynecological cancer patients.

\section{Acknowledgements}

The authors would like to thank Dr Tetsuji Kurokawa, Dr Yoko Chino and Dr Akiko Shinagawa (all University of
Fukui, Fukui, Japan) for their advice on data analysis and assistance with the study.

\section{Funding}

The present study was partly supported by a Grant-in-Aid for Scientific Research from the Japan Society for the Promotion of Science (grant no. 18H02944).

\section{Availability of data and materials}

The datasets used and/or analyzed during the current study are available from the corresponding author on reasonable request.

\section{Authors' contributions}

KK, MY, and YY wrote the manuscript. KK, MY, MAS, MS, YU, YA and YY collected and analyzed data, and edited the manuscript. All authors read and approved the final manuscript.

\section{Ethics approval and consent to participate}

The present study was reviewed and approved by the institutional review boards of University of Fukui, Yokohama City University Graduate School of Medicine, Osaka University Graduate School of Medicine and University of the Ryukyus before the start of the present study. Informed consent was obtained in the form of an opt-out option on the website.

\section{Patient consent for publication}

Not applicable.

\section{Competing interests}

The authors declare that they have no competing interests.

\section{References}

1. Annual Report on the Aging Society: 2018 (Summary). Cabinet Office, Tokyo, 2018.

2. Gawande AA, Kwaan MR, Regenbogen SE, Lipsitz SA and Zinner MJ: An apgar score for surgery. J Am Coll Surg 204: 201-208, 2007.

3. Bilimoria KY, Liu Y, Paruch JL, Zhou L, Kmiecik TE, Ko CY and Cohen ME: Development and evaluation of the universal ACS NSQIP surgical risk calculator: A decision aid and informed consent tool for patients and surgeons. J Am Coll Surg 217: 833-842, 2013.

4. Szender JB, Frederick PJ, Eng KH, Akers SN, Lele SB and Odunsi K: Evaluation of the national surgical quality improvement program universal surgical risk calculator for a gynecologic oncology service. Int J Gynecol Cancer 25: 512-520, 2015.

5. Rivard C, Nahum R, Slagle E, Duininck M, Vogel RI and Teoh D: Evaluation of the performance of the ACS NSQIP surgical risk calculator in gynecologic oncology patients undergoing laparotomy. Gynecol Oncol 141: 281-286, 2016.

6. Pecorelli S: Revised FIGO staging for carcinoma of the vulva, cervix, and endometrium. Int J Gynaecol Obstet 105: 103-104, 2009.

7. Prat J; FIGO Committee on Gynecologic Oncology: Staging classification for cancer of the ovary, fallopian tube, and peritoneum. Int J Gynaecol Obstet 124: 1-5, 2014.

8. Prat J: FIGO staging for uterine sarcomas. Int J Gynaecol Obstet 104: 177-178, 2009.

9. Charlson ME, Pompei P, Ales KL and MacKenzie CR: A new method of classifying prognostic comorbidity in longitudinal studies: Development and validation. J Chronic Dis 40: 373-383, 1987. 
10. Quan H, Li B, Couris CM, Fushimi K, Graham P, Hider P, Januel JM and Sundararajan V: Updating and validating the charlson comorbidity index and score for risk adjustment in hospital discharge abstracts using data from 6 countries. Am J Epidemiol 173: 676-682, 2011.

11. Kobayashi K, Imagama S, Ando K, Inagaki Y, Suzuki Y, Nishida Y, Nagao Y and Ishiguro N: Analysis of falls that caused serious events in hospitalized patients. Geriatr Gerontol Int 17: 2403-2406, 2017.

12. Katayama H, Kurokawa Y, Nakamura K, Ito H, Kanemitsu Y, Masuda N, Tsubosa Y, Satoh T, Yokomizo A, Fukuda H and Sasako M: Extended clavien-dindo classification of surgical complications: Japan clinical oncology group postoperative complications criteria. Surg Today 46: 668-685, 2016.

13. Regenbogen SE, Ehrenfeld JM, Lipsitz SR, Greenberg CC, Hutter MM and Gawande AA: Utility of the surgical apgar score: Validation in 4119 patients. Arch Surg 144: 30-36, 2009.

14. Zighelboim I, Kizer N, Taylor NP, Case AS, Gao F, Thaker PH, Rader JS, Massad LS, Mutch DG and Powell MA: 'Surgical Apgar Score' predicts postoperative complications after cytoreduction for advanced ovarian cancer. Gynecol Oncol 116: 370-373, 2010

15. Clark RM, Lee MS, Rauh-Hain JA, Hall T, Boruta DM, del Carmen MG, Goodman A, Schorge JO and Growdon WB: Surgical apgar score and prediction of morbidity in women undergoing hysterectomy for malignancy. Gynecol Oncol 136: 516-520, 2015.

16. Kurata K, Chino Y, Shinagawa A, Kurokawa T and Yoshida Y: Surgical apgar score predicts 30-day morbidity in elderly patients who undergo non-laparoscopic gynecologic surgery: A retrospective analysis. Int J Surg 48: 215-219, 2017.

17. World Health Organization (WHO): World Alliance for Patient Safety: forward programme 2008-2009, 1st edition. WHO, Geneva, 2008. https://apps.who.int/iris/handle/10665/70460. Accessed June 25, 2008.

18. Park SH, Lee JY, Nam EJ, Kim S, Kim SW and Kim YT: Prediction of perioperative complications after robotic-assisted radical hysterectomy for cervical cancer using the modified surgical apgar score. BMC Cancer 18: 908, 2018.

19. Uccella S, Bonzini M, Palomba S, Fanfani F, Malzoni M Ceccaroni M, Seracchioli R, Ferrero A, Berretta R, Vizza E, et al: Laparoscopic vs. open treatment of endometrial cancer in the elderly and very elderly: An age-stratified multicenter study on 1606 women. Gynecol Oncol 141: 211-217, 2016.
20. Berretta R, Merisio C, Melpignano M, Rolla M, Ceccaroni M, DE Ioris A, Patrelli TS and Nardelli GB: Vaginal versus abdominal hysterectomy in endometrial cancer: A retrospective study in a selective population. Int J Gynecol Cancer 18: 797-802, 2008.

21. Goldman LS: Medical illness in patients with schizophrenia J Clin Psychiatry 60 (Suppl 21): S10-S15, 1999.

22. Liperoti R, Pedone C, Lapane KL, Mor V, Bernabei R and Gambassi G: Venous thromboembolism among elderly patients treated with atypical and conventional antipsychotic agents. Arch Intern Med 165: 2677-2682, 2005.

23. Parker C, Coupland C and Hippisley-Cox J: Antipsychotic drugs and risk of venous thromboembolism: Nested case-control study. BMJ 341: c4245, 2010.

24. Daumit GL, Pronovost PJ, Anthony CB, Guallar E, Steinwachs DM and Ford DE: Adverse events during medical and surgical hospitalizations for persons with schizophrenia. Arch Gen Psychiatry 63: 267-272, 2006.

25. Tinquaut F, Freyer G, Chauvin F, Gane N, Pujade-Lauraine E and Falandry C: Prognostic factors for overall survival in elderly patients with advanced ovarian cancer treated with chemotherapy: Results of a pooled analysis of three GINECO phase II trials. Gynecol Oncol 143: 22-26, 2016.

26. Ferrero A, Villa M, Tripodi E, Fuso L and Menato G: Can vulnerable elders survey-13 predict the impact of frailty on chemotherapy in elderly patients with gynaecological malignancies? Medicine (Baltimore) 97: e12298, 2018.

27. Suh DH, Kim JW, Kim HS, Chung HH, Park NH and Song YS: Pre- and intra-operative variables associated with surgical complications in elderly patients with gynecologic cancer: The clinical value of comprehensive geriatric assessment. J Geriatr Oncol 5: 315-322, 2014

28. Ahmed A, Deng W, Tew W, Bender D, Mannel RS, Littell RD, DeNittis AS, Edelson M, Morgan M, Carlson J, et al: Pre-Operative assessment and post-operative outcomes of elderly women with gynecologic cancers, primary analysis of NRG CC-002: An NRG oncology group/gynecologic oncology group study. Gynecol Oncol 150: 300-305, 2018.

This work is licensed under a Creative Commons Attribution-NonCommercial-NoDerivatives 4.0 International (CC BY-NC-ND 4.0) License. 